\title{
Rho-Kinase Inhibitors as a novel medication for Glaucoma Treatment - A Review of the literature
}

\author{
Author: Asaad A. Ghanem', Tharwat Mokbel ${ }^{1}$, Ahmed A. Ghanem², Amgad El Nokrashy' \\ 1- Mansoura Ophthalmic Center, Mansoura University, Egypt. \\ 2- Faculty of medicine, Mansoura University, Egypt.
}

Corresponding author: Prof. Dr. Asaad A. Ghanem; Mansoura Ophthalmic Center, Faculty of Medicine, Mansoura University, Mansoura, Egypt. PO. 35516 Te: 00201117437876; Email: asaadghanem@hotmail.com

Received: 11-1-2021, Accepted: 3-3-2021, Published online:10-6-2021

EJO(MOC) 2021;2:110-120.

Running title: Rho-Kinase Inhibitors - Review

\begin{abstract}
Glaucoma is a progressive optic neuropathy, characterized by loss of retinal ganglion cells with subsequent deterioration in visual field. A major clinically modifiable risk factor for glaucomatous deterioration is elevated intraocular pressure (IOP) as a sequence of an increased aqueous humor (AH) outflow resistance. Aside from the introduction of prostaglandin analogs, topical carbonic anhydrase inhibitors, and topical alpha agonists in the 1990s, a new pharmaceutical agent to lower IOP have been introduced approximately 20 years.

Rho-kinase (ROCK) Inhibitors, a new pharmacological class of hypotensive glaucoma medication targeting specifically the diseased trabecular outflow pathway. Rock inhibitors function by relaxing the trabecular meshwork, which subsequently leads to improved aqueous humor outflow and lower IOP. ROCK is a downstream effector of a small-G protein Rho.

In addition to improving the outflow facility of the trabecular meshwork, Rho kinase inhibitors also enhance retinal ganglion cell survival after ischemic injury and increase ocular blood flow. ROCK inhibitors were reported to block TGF-B myofibroblast differentiation of human tendon fibroblasts, suggesting that may reduce postoperative conjunctival scarring after glaucoma surgery

The objectives of this review are to describe the basic science underlying the mechanism of ROCK inhibitors as a therapy to lower IOP, their neuro-protective, and vasoactive properties.

Keywords: Glaucoma, Intraocular Pressure, Rho Kinase Inhibitors, Aqueous outflow, Trabecular Meshwork, Neuroprotection.
\end{abstract}

\section{Introduction}

Glaucoma is a progressive optic neuropathy, characterized by loss of retinal ganglion cells and their axons, resulting in a characteristic and distinctive appearance of the optic disc cupping, concomitant to visual loss, which is the second leading cause of blindness in the world ${ }^{1,2}$. The disease affected more than 60.5 million individuals in 2010 and is projected to reach 79.6 million by $2020^{2}$. Glaucoma is almost always asymptomatic, especially in the early stages, and that is one of the reasons why glaucoma remains undiagnosed in up to half of total cases in developed nations, and even more in regions of the developing world ${ }^{3}$.

A major risk factor for glaucomatous visual field loss is elevated intraocular pressure (IOP $)^{4}$. A study have shown that lowering IOP reduces the risk of glaucomatous progression ${ }^{5}$.

Egyptian Journal of Ophthalmology, a publication of Mansoura Ophthalmic Center.

Address: Mansoura Ophthalmic Center, Mansoura University, Mansoura, Egypt.

Tel. 0020502202064. Fax. 0020502202060.

E-mail: ejo_moc@yahoo.com; tharwatmokbel@yahoo.com 
There are five mainly class of eye drops for IOP reduction: cholinergic, $\beta$-blockers, $\alpha$-agonists, carbonic anhydrase inhibitors and prostaglandins analogs. These medications in monotherapy do not control IOP efficiently in some patients, and approximately half of patients with elevated IOP are treated by coadministration of 2 or more glaucoma medications. In addition, all these class of eye drops present ocular and systemic undesirable effects that can impair the treatment. For these reasons, researches searching for new IOP-lowering drugs ${ }^{5}$.

Currently, the available pharmacological treatment for glaucoma lowers the IOP either via decreasing the aqueous humor production by the ciliary process (i.e., $\beta$-blockers agents; carbonic anhydrase inhibitors; alpha agonists), or improving aqueous outflow through the unconventional pahtway (i.e., prostaglandins analogues) ${ }^{6}$. However, there are no pharmacological agents targeting specifically the diseased trabecular outflow pathway. Combinations with other glaucoma agents, new molecular entities as ROCK inhibitors will be reported as new biological approaches to affect the Rho/ROCK pathway ${ }^{7}$.

Small-molecule inhibitors of ROCK are a new class of drug that have been recently studied as potential glaucoma therapeutics. The Rho kinase inhibitors represent a new class of glaucoma medications that inhibit the downstream pathway of the Rho family of small G-proteins to increase outflow from the conventional (trabecular) outflow pathway in the eye. Several of these Rho kinase inhibitors, ripasudil and netarsudil, have recently reached the market and are used in clinical practice in several countries ${ }^{8}$.

IOP lowering in rabbits eyes using a selective ROCK inhibitor Y-27632 was first described by Honjo et al ${ }^{9}$. Rock inhibitors reduce IOP by increasing aqueous humor drainage through the trabecular meshwork. In addition to improving the outflow facility of the trabecular meshwork, Rho kinase inhibitors also enhance retinal ganglion cell survival after ischemic injury ${ }^{10}$ and increase ocular blood flow ${ }^{11}$. Because of these functions, ROCK inhibitors have recently gained interest for several ongoing clinical trials.

\section{METHODS:}

A literature conducted a detailed review using various electronic databases, including PubMed, Science Direct, and Journals of Ophthalmology. The dates used to define the search were between January 2010 and October, 2019. For the PubMed search, Medical Subject Headings (MeSH) were used. The principal term used to dictate the MeSH search was "Rho kinase inhibitor". It was connected to the following terms : glaucoma, intraocular pressure, trabecular meshwork, neuroprotection. Multiple clinical studies were identified and reviewed. Sources from these studies were identified, reviewed. Non-english literatures were excluded.

\section{Review Of The Literature}

\section{I-Physiology of the aqueous humor outflow}

The balance between the inflow and outflow of aqueous humor is responsible for IOP. There are two aqueous humor outflow pathways in the eye. Under normal conditions, the trabecular meshwork (TM) is responsible for the majority of the aqueous humor outflow. An auxiliary "unconventional" uveoscleral pathway exists, through the iris root and ciliary muscle, but it carries less than $10 \%$ of the total flow in the eye ${ }^{12}$.

The TM can be subdivided in three regions: the uveal meshwork, corneoscleral meshwork and juxtacanalicular connective tissue (JCT). After passing through the TM, the aqueous humor drains sequentially through the Schlemm's canal (SC), collector channels, and later to the systemic circulation through the episcleral veins. An increased resistance to flow in the conventional pathway $(90 \%$ of total aqueous humor outflow) is predominantly responsible for elevated IOP in the many types of glaucoma. In primary open-angle glaucoma (POAG), the most common type, increased outflow resistance has been classically associated with the juxtacanalicular portion of the TM mediated through extracellular matrix, and in the endothelial-lined $\mathrm{SC}^{13}$.

Histologically, TM is a complex tissue consisting of TM cells, extracellular matrix (ECM), and empty spaces, through which aqueous humor runs. These spaces gradually become smaller as they become closer to SC, and the density of TM cell and ECM is relatively high in this zone, resulting in a 
higher resistance to flow. The functional properties of the TM are often affected in $\mathrm{POAG}^{14}$.

Conventional outflow pathway influenced by the contraction of the ciliary muscle when exposed to a muscarinic agent such as pilocarpine. When the ciliary muscle contracts, its insertions widen the intercellular spaces in the TM and the permeability of the tissue increases; subsequently, uveoscleral outflow decreases. Studies have shown that the TM is also thought to have a dynamic regulatory mechanism because of its smooth muscle-like properties, as evidenced by the expression of $\alpha$-smooth muscle actin and myosin, ion channels and G-protein coupled receptors (GPCR) in TM cells ${ }^{15,16}$.

Moreover, physiological contractile agonists, cytokines, growth factors, pharmacological agents, have been shown to affect the contractile and relaxation properties of TM tissue. Based on perfusion studies including anterior segments of the eye, the TM alone has been shown to participate in the regulation of aqueous humor outflow through $\mathrm{SC}^{17}$.

The actomyosin system, composed of the contractile proteins actin and myosin, is responsible for the regulating contraction and relaxation in muscle tissues, which included in the TM and JCT/SC (Fig. 1) ${ }^{30}$. Relaxation of the TM would relax the cellular actomyosin system resulting in cellular relaxation and/or cell shape alteration, which subsequently increase intercellular space leading to increased aqueous outflow ${ }^{18}$.

Aqueous humor biomarkers such as transforming growth factor $\beta$ (TGF- $\beta$ ) concentration is higher in glaucomatous patients than in healthy controls, and TGF- $\beta$ is believed to play an important role in the pathogenesis of glaucoma ${ }^{19}$. Other bioactive endogenous mediators of contractility molecules, such as cytokines and endothelin are also present in higher concentrations in glaucomatous eyes ${ }^{20}$.
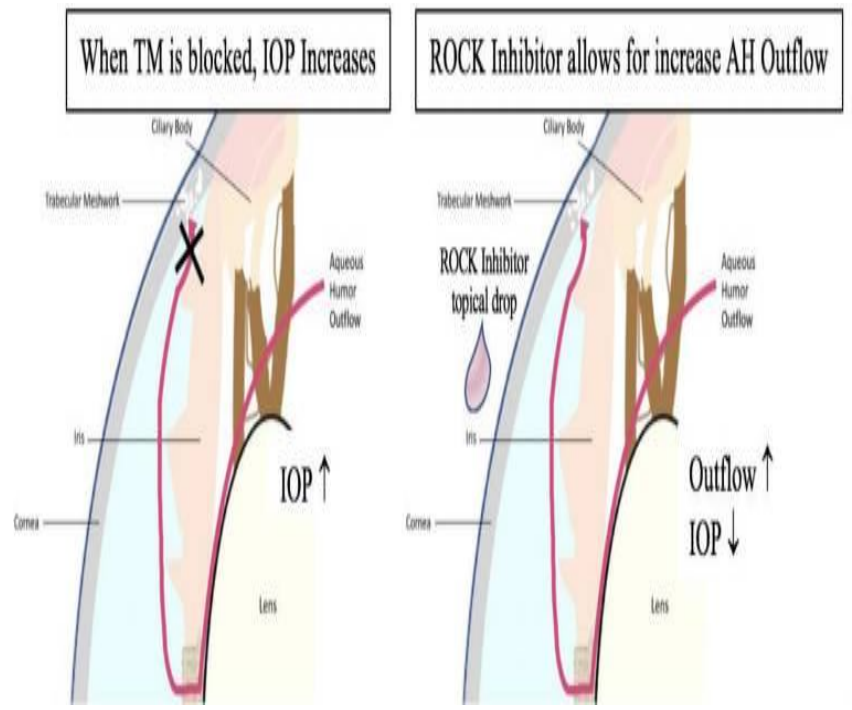

Fig. 1: Schematic View of the Treatment of Glaucoma using ROCK Inhibitor Drops TM: Trabecular Meshwork; IOP: Intraocular Pressure; ROCK inhibitor: Rho kinase inhibitor; AH: Aqueous Humor ${ }^{30}$.

\section{II- Rho Kinase signaling in Smooth Muscle Contraction}

The regulation of TM contractility is controlled by both calcium-dependent and independent mechanisms. Smooth muscle contraction is regulated predominantly by the phosphorylation of myosin light chain (MLC). MLC is phosphorylated by calcium/calmodulin-dependent MLC kinase (MLCK) and dephosphorylated by calciumindependent MLC phosphatase (MLCP) ${ }^{21}$. This regulation by $\mathrm{Ca}^{++}$concentration, modulation of MLC phosphorylation can occur through signaling pathways, such as the Rho/Rho kinase pathway.

Downstream effectors of Rho include the ROCKs that are protein serine/threonine kinases. Structurally, ROCKs are composed of three major constitutes: an $\mathrm{N}$ terminal kinase domain that phosphorylates protein targets, a C-terminal auto inhibitory domain that decreases kinase activity via intermolecular interactions, and a coiled-coil Rho-binding domain which facilitate the switch from the inactive to active conformation. ROCKs can phosphorylate the same serine residue of MLC phosphorylated by $\mathrm{MLCK}^{22}$, in a manner independent of calcium concentrations which leads to enhanced smooth muscle contraction. 


\section{III- Rho Kinase Inhibitors modulate the cytosteleton}

The Rho family (RhoA, RhoB, RhoC) are small Gproteins that are active when bound to guanosine triphosphate and inactive when bound to guanosine diphosphate. They are activated by a number of secreted cytokines including endothelin-1, thrombin, agiontension II, lysophophatidic acid, and transforming growth factor (TGF)- $\beta$, or via integrin activation. They regulate cell morphology, polarity, proliferation, adhesion, motion, cytokinesis, and apoptosis along with smooth muscle contraction and neurite elongation $^{23}$

The effectors of the Rho family are the Rho kinases, ROCK1 and ROCK2, both are serine/threonine kinase isoforms. ROCK1 and ROCK2 contain an N-terminal kinase domain that phosphorylates target proteins, followed by cysteine-rich autoinhibitory domain toward the $\mathrm{C}$ terminus that limits kinase activity via intramolecular interactions ${ }^{24}$. ROCK1 and ROCK2 have a similar structure with $65 \%$ overall homology, and $87 \%$ identity in the kinase domain, indicating that both isoforms can activate the same targets but allows for some differences in effect (Fig. 2) ${ }^{30}$. Their genes are located on chromosome 18 (18q11.1) and chromosome 2 (2p24), respectively ${ }^{25}$.

It is known that TM and ciliary muscle tissue express many components of the Rho signaling pathway such as ROCK1 and ROCK2, RhoA, MLC, MLCK and MLCP. For this reason the activity of ROCK through the Rho signaling pathway is thought to be essential for regulating the cellular morphology and contractility of the conventional aqueous outflow pathway. Rho kinase inhibitors are the first class of glaucoma drugs that increase aqueous humor outflow by working directly on the TM cell and SC cell, lowering IOP. Different Rho kinase inhibitors (Y-27632, H-1152 and fasudil) induced rapid and long-lasting IOP lowering in various animal models, including rabbit and monkey ${ }^{26}$.

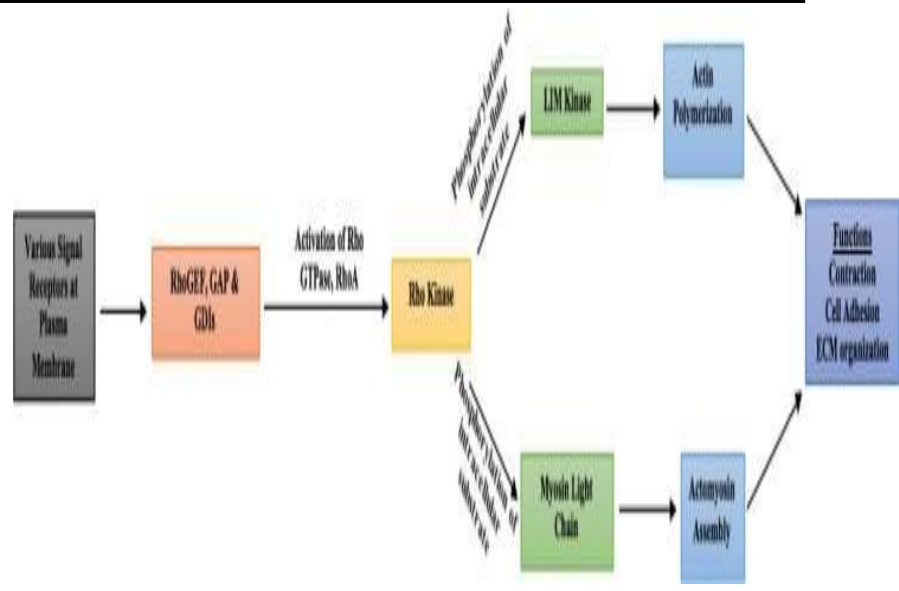

Fig. 2: A Schematic View of Rho Kinase Involved Metabolic Pathway. RhoGEF: Rho Guanine nucleotide Exchange Factor; GAP: GTPase Activating Proteins; GDI: Guanine nucleotide Disassociation Inhibitor; RhoA: Ras Homolog Gene Family Member A; ECM: Extracellular Matrix; GTPase: Guanosine triphosphatase ${ }^{30}$

\section{IV- Rho-kinase inhibitors; mechanism of action:}

The mechanism by which Rho-kinase inhibitors increase aqueous outflow facility through conventional pathway is hypothesized that by inhibiting ROCK, leading to disruption of specific components of the cellular cytoskeleton and diminish the contractile tone of the conventional outflow pathway tissues. The effect of these two mechanisms will increase paracellular fluid flow through the inner wall of the $\mathrm{SC}$, stemming from altered cell shape and cell junctions and the relaxation of the TM and JCT, which will lead to an increase in outflow facility and a lowering of IOP.

A number of mechanisms have been proposed as to how modifying the cytoskeleton can alter outflow resistance. A hypothesis proposed that Schlemm's canal cell stiffness modulates aqueous humor outflow resistance by affecting the propensity of these cells to form pores ${ }^{27}$. Where, less stiff cells are able to form more pores, thus decreasing the funneling effect and decreasing outflow resistance. Other hypothesis proposed that changes in the Schlemm's canal cell cytoskeleton leading to changes in focal adhesions thereby releasing the attachments between the Schlemm's canal cells and the JCT, expanding the spaces in the JCT and decreasing the magnitude of the funneling effect ${ }^{28}$. 
V- Rho-kinase inhibitors formula

A- Ripasudil (K-115)

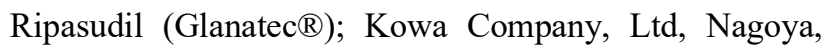
Aichi, Japan) was approved in Japan for the treatment of primary OAG and ocular hypertension in September 2014. Ripasudil hydrochloride hydrate was originally discovered by D. Western Therapeutics Institute and developed for the treatment by Kowa Company, $\operatorname{Ltd}^{\underline{29}}$. It has the chemical formula of $\mathrm{C} 15 \mathrm{H} 18 \mathrm{FN} 3 \mathrm{O} 2 \mathrm{~S}$ and has the International Union of Pure and Applied Chemistry (IUPAC), name being 4fluoro-5- (((2S)- 2-methy 1-1,4- diazepan-1 -yl) sulfonyl) isoquinoline $\mathrm{e}^{30}$.

Ripasudil 0.4\% BID approved as a clinically useful concentration and dosing frequency for the treatment of glaucoma and OHT. The $0.4 \%$ solution lowered IOP on average by $2-4.4 \mathrm{mmHg}$ two hours after instillation and continued to deliver statistically significant pressure reduction for at least 7 hours.

The clinical trials demonstrated the dose-dependent and transient nature of conjunctival hyperemia associated with its use. A study specifically designed to investigate the timecourse of ripasudil-induced conjunctival hyperemia found peak intensity at 15 minutes after instillation and a gradual return to baseline at 120 minutes. The study found that conjunctival hyperemia (76\%), blepharitis $(21 \%)$, and allergic conjunctivitis (20\%) were the most commonly reported adverse events attributed to ripasudil monotherapy. Temporaryc orneal guttae-like findings were reported and may be related to their potential use for corneal clearing as a salvage agent in patients with Fuchs dystrophy undergoing various types of keratoplasty31. Rho kinase inhibitors can cause vasodilation via relaxation of vascular smooth muscle, which is likely the cause of the common adverse effect of conjunctival hyperemia.

As ripasudil was primarily evaluated as an adjunctive agent for use in combination with commonly used first-line agents. A study reported the additive IOP-lowering efficacy of ripasudil $0.4 \%$ BID with either timolol $0.5 \%$ BID or latanoprost $0.005 \%$ QD in patients with POAG or OHT32. Treatment with ripasudil resulted in a lower mean IOP in the timolol group as the additive effect was $0.9 \mathrm{mmHg}$ at trough and $1.6 \mathrm{mmHg}$ at peak. In the latanoprost group, there was no significant difference compared to placebo at trough; at peak, ripasudil resulted in an additional $1.4 \mathrm{mmHg}$ reduction.

In one of the retrospective studies, there was no significant additional IOP reduction in the subgroup of patients defined as having normal-tension glaucoma ${ }^{33}$. Two retrospective studies suggest it is safe to use ripasudil to lower IOP in eyes with ocular hypertensive eyes with uveitis $^{34,35}$.

Ripasudil has been speculated to have a potential role in neuroprotection, as in vivo and in vitro mouse studies have shown that oral administration of the drug delayed retinal ganglion cell death, possibly because of some role in suppression of reactive oxygen species ${ }^{36}$.

\section{B- Netarsudil (AR-13324)}

Netarsudil $\left(\right.$ Rhopressa $\left.^{\circledR}\right)$, is not only a Rho kinase inhibitor but also functions as a norepinephrine transporter inhibitor. It was developed by (Aerie Pharmaceuticals, Bedminster, NJ,US) as one of a class of amino-isoquinoline amide Rho kinase inhibitors. It was approved for use in the United States to treat glaucoma in late 2017. Netarsudil, known as AR-13503 in clinical trials. It has the chemical formula of $\mathrm{C} 28 \mathrm{H} 27 \mathrm{~N} 3 \mathrm{O} 3$ and has the IUPAC name of (4((1S) -1- (Aminomethyl) -2- (isoquinolin -6- ylamino) -2oxoethyl) pheny) methyl2, 4-dimethylbenzoate ${ }^{37}$.

Netarsudil has been shown to decrease IOP within two hours of instillation and also to sustain this decrease for a $24-$ hour period after dosing. Netarsudil uses two mechanisms to lower IOP; by acting as both a ROCK inhibitor and a norepinephrine transport inhibitor. The latter helps to prolong reduction in IOP by constriction of vascular structures in the eye. This decreases episcleral venous pressure and reduces blood flow to the ciliary processes, inhibiting production of aqueous humor ${ }^{38}$.

A study compared the ocular hypotensive efficacy of netarsudil 0.01\% QD, netarsudil 0.02\% QD and latanoprost $0.005 \%$ QD in patients with OHT or POAG with baseline IOP $\geq 24 \mathrm{mmHg}$ and $<36 \mathrm{mmHg}$ after washout. Mean baseline IOP was about $25.5 \mathrm{mmHg}$. IOP lowering observed on day 28 was similar to that on day 14 and was found to be 
5.5, 5.7 and $6.8 \mathrm{mmHg}$ in the netarsudil $0.01 \%$, netarsudil $0.02 \%$ and latanoprost $0.005 \%$ groups, respectively. Neither concentration of netarsudil was as effective as latanoprost. Netarsudil was therefore thought to be relatively more effective in patients with lower baseline IOP, possibly due to its ability to lower episcleral venous pressure ${ }^{39}$. Conjunctival hyperemia, most commonly graded as mild, occurred in 52\%, $57 \%$ and $15 \%$ of subjects in the netarsudil $0.01 \%$, netarsudil $0.02 \%$ and latanoprost $0.005 \%$ groups, respectively. Increased lacrimation (6\%), subconjunctival hemorrhage $(5 \%)$ were also reported in the two netarsudil groups.

Netarsudil is likely most effective as an adjunctive agent for patients already using other classes of IOP-lowering medication and may be helpful as a first-line therapy for patients with normal tension glaucoma, as it has been shown to be more effective for patients with lower baseline IOPs.

\section{C-Latanoprost/Netarsudil}

Netarsudil $0.02 \%$ has been used in a fixed-dose combination (FDC) with latanoprost $0.005 \%$. This fixed-dose combination was very recently approved by the US FDA in 2019 as Roclatan ${ }^{\mathrm{TM}}$ (PG324) (Aerie Pharmaceuticals).

In a phase II clinical trial over 28 days, this FDC was statistically superior to either of the two drugs alone. The FDC (with netarsudil at $0.02 \%$ ) has significant increased IOP lowering of $-1.9 \mathrm{mmHg}$ compared with latanoprost and $-2.6 \mathrm{mmHg}$ compared with netarsudil alone ${ }^{40}$. Adverse events were similar to those previously reported with netarsudil, with the most common event being mild conjunctival hyperemia.

To evaluate netarsudil as an adjunctive agent in combination with latanoprost, a 28-day randomized, controlled clinical trial evaluated the fixed combination of netarsudil (at concentrations of $0.01 \%$ and $0.02 \%$ ) and latanoprost $0.005 \%$ dosed once-daily. The mean diurnal efficacy of the fixed combination formulated with a concentration of netarsudil $0.02 \%$ was statistically superior to each of its components alone by a margin of $2.6 \mathrm{mmHg}$ vs. netarsudil and $1.9 \mathrm{mmHg}$ vs. latanoprost (each agent was dosed once-daily) ${ }^{41}$.

\section{D-AR-12286}

AR-12286 was developed by Aerie Pharmaceuticals by screening a collection of water soluble amino-isoquinoline amides to find those that were both stable and active in affecting the shape of trabecular meshwork cells ${ }^{42}$.

A phase 1 study in normal subjects of AR-12286 0.5\% for 8 days reported significant IOP-lowering with an average maximum decrease of approximately $7 \mathrm{mmHg}$, however, adverse effects including conjunctival hyperemia, ocular irritation, increased lacrimation, and blurred vision were reported $^{43}$. A larger, placebo-controlled randomized phase 2 clinical trial in patients with POAG or OHT evaluated AR12286 at a lower maximum concentration $(0.25 \%)$ over a 3 week study period showed a maximum average pressure reduction of approximately $4.5 \mathrm{~mm} \mathrm{Hg}$, as compared to placebo $^{44}$.

\section{E-SNJ-1656 (previously known as Y-39983)}

SNJ-1656, developed by Senju Pharmaceutical Co, was the first Rho kinase inhibitor studied in a clinical trial to lower IOP. It is thirty times more effective in inhibiting Rho kinase activity than Y-27632, and in animal studies, topical administration of SNJ-1656 resulted in large reductions in outflow resistance and IOP.

The phase 1 study evaluated the ocular hypotensive efficacy and safety compared to the vehicle in healthy subjects after a single instillation and after 7 days of repeated (QD or BID) instillation. Peak IOP reduction was recorded at 4 hours after instillation, $3.0 \pm 1.2 \mathrm{~mm} \mathrm{Hg}$ with the highest concentration tested $(0.1 \%)$. Conjunctival hyperemia was observed in all patients but resolved in most cases within 24 hours after a single instillation ${ }^{45}$.

The phase 2 study evaluated various concentrations of SNJ-1656 (0.03\% to $0.1 \%)$ for 7 days in patients with POAG and OHT. The relative IOP reduction compared to placebo from a baseline of about $22 \mathrm{mmHg}$ was $3-3.5 \mathrm{mmHg}$ at peak ( 2 hours after instillation of the morning dose) and 2 $\mathrm{mmHg}$ at trough (prior to instillation of the morning dose). Mild to moderate conjunctival hyperemia occurred in about $60 \%$ of subjects ${ }^{45}$. 


\section{F- Others Rho kinase inhibitors in development}

INS-117548 (Merck Sharp and Dohme, Kenilworth, NJ, USA) has completed a phase I clinical trial (NCT00767793) but is no longer in development. Notably, Rho kinase inhibitor AR-12286 (Aerie Pharmaceuticals) completed phase II clinical trials but is no longer in development ${ }^{46}$.

\section{VI- Other applications of Rho kinase inhibitors}

Several studies have shown that Rho kinase inhibitors might also benefit glaucoma patients by improving ocular blood flow as well as presenting potential neuroprotective effects, besides possibly preventing postoperative scarring after glaucoma filtration surgery ${ }^{47}$. Rho kinase inhibitors increase blood flow in systemic hypertensive conditions through relaxation of vascular endothelial smooth muscle. As altered ocular blood flow is believed to participate in the pathophysiology of certain types of glaucoma, Rho kinase inhibitors could provide additional benefit as therapeutics agents for glaucoma.

In POAG, it is widely thought that the initial site of neuronal injury is the retinal ganglion cell axon at the level of the lamina cribrosa. Rho kinase signaling is critical in axonal development, maintenance and regeneration1. Its role is exerted through its regulation of many elements of the axonal cytoskeleton, including actin, microtubules and intermediate filaments as well as through regulation of inflammation mediated by activation of $\mathrm{NF}-\kappa \mathrm{B}^{48}$.

Significantly elevated levels of RhoA have been found in the optic nerve head of POAG eyes as compared with agematched controls, supporting a possible role for Rho in glaucomatous neuropathy ${ }^{49}$.

ROCK inhibitors have also been shown to play a role in neuron survival and axon regeneration. TGF- $\beta$ is an important cytokine involved in the regulation of post-surgical wound healing and scar formation in the setting of glaucoma surgery. ROCK inhibitors were reported to block TGF-B myofibroblast transdifferentiation of human tendon fibroblasts, suggesting that ROCK inhibitors may reduce postoperative conjunctival scarring after glaucoma filtration surgery $^{50}$.

\section{VII- ROCK adverse effects}

It has been shown that ROCK inhibitors are potent vasodilators, including the conjunctival vasculature. Arnold et $\mathrm{al}^{51}$ studied the effects of Y-27632 on the intraocular penetration of timolol maleate, and they found that ROCK inhibitors reduced the intraocular penetrations of timolol, presumably due to increased systemic elimination through the conjunctival vasculature.

Bacharach J. et $a l^{52}$ evaluated the ocular hypotensive efficacy and safety of AR-13324 ophthalmic solution compared with a control group using latanoprost, in patients with open-angle glaucoma (OAG) or ocular hypertension $(\mathrm{OH})$. Patients were randomized to receive AR-13324 ophthalmic solution $0.01 \%$, daily (pm), AR-13324 ophthalmic solution $0.02 \%$ daily (pm), or latanoprost $0.005 \%$ daily (pm) for 28 days. Their results showed that AR-13324 $0.02 \%$ was less effective than latanoprost by approximately 1 $\mathrm{mmHg}$ and the major safety finding was ocular hyperemia, which was more common for both concentrations of AR13324 than for latanoprost.

Tanihara $H$. et $a l^{53}$ studied the dose dependency and safety of the Rho kinase inhibitor K-115. 210 patients with primary $\mathrm{OAG}$ or $\mathrm{OH}$ were subdivided into 4 groups and were treated with $\mathrm{K}-115$ in concentrations of $0.1 \%, 0.2 \%$, and $0.4 \%$ or placebo twice daily for 8 weeks. The dose response of IOP reduction and the incidence of adverse events by K115 or placebo were investigated. The mean IOP reduction was $-3.1 \mathrm{~mm} \mathrm{Hg}$ at 8 hours after instillation in the $0.4 \%$ group, and all groups presented mild conjuntival hyperemia. The ROCK inhibitor SNJ-1656 was also demonstrated to be a safe hypotensive topical agent in human eyes.

\section{CONCLUSION}

Unlike all other glaucomatous topical drugs, Rho kinase inhibitors target the trabecular pathway and act to increase aqueous humor outflow. This review has also shown that they enhance ocular blood flow, present neuroprotective effects and they may inhibit scarring after glaucoma filtering surgery. This article also presents the newest clinical trials published in the literature, updating the current status of these drugs for use in glaucomatous eyes. 
Several studies demonstrated that ROCK inhibitors provided clinically and statistically significant ocular hypotensive efficacy in patients with ocular hypertension and glaucoma. However, it remains to be seen in future clinical trials how adverse side effects, such as conjunctival hyperemia, would lower patient compliance. Also it would be necessary to know if ROCK inhibitors could be used synergistically with other IOP-lowering drugs.

Because their primary mechanism of action is different from other ocular hypotensive medications, in that they act to normalize outflow resistance, Rho kinase inhibitors were developed with the hope that in addition to their use in monotherapy, they could provide additional IOP reduction when used with other ocular hypotensive agents. Although these agents have been shown to be effective in lowering IOP, both as monotherapy and adjunctively with betablockers and prostaglandin analogs, their side effect profile raises serious concerns about the likelihood of their acceptance by patients.

It is emphasized that laboratory evidence suggests Rho kinase inhibitors may have neuroprotective activity and might improve ocular blood flow. It would be ideal to have a drug that not only lowers IOP but also protects retinal ganglion cells from IOP-independent factors that contribute to disease progression.

\section{Corresponding author}

Correspondence to Asaad A. Ghanem

Email: asaadghanem@hotmail.com

\section{Affiliations}

Asaad Ahmed Ghanem

Mansoura Ophthalmic Center, Mansoura University, Mansoura, Egypt.

\section{Ethics declarations}

\section{Conflict of interest}

Asaad A. Ghanem, Tharwat Mokbel, Ahmed A. Ghanem, Amgad El Nokrashy, all authors have no conflicts of interest that are directly relevant to the content of this review.

Funding: No sources of funding were used to conduct this review.
Reviewer disclosures: No relevant financial or other relationships to disclose.

Declaration of interest: No financial affiliations or financial involvement with any organization or entity with a financial competing with the subject matter or materials discussed in the review.

\section{REFERENCES}

1- Weinreb RN, Khaw PT. Primary open-angle glaucoma. Lancet 2004; 363 (9422): 1711-1720.

2- Quigley HA, Broman AT. The number of people with glaucoma worldwide in 2010 and 2020. Br J Ophthalmol. 2006; 90(3):262-267.

3- Quigley HA. Glaucoma. Lancet. 2011;377(9774):13671377.

4- The AGIS Investigators. The Advanced Glaucoma Intervention Study (AGIS): 7. The relationship between control of intraocular pressure and visual field deterioration. Am J Ophthalmol. 2000;130(4):429-440.

5- Heijl A, Leske MC, Bengtsson B, Hyman L, Hussein M. Reduction of intraocular pressure and glaucoma progression: results from the early manifest glaucoma trial. Arch Ophthalmol. 2002; 120(10):1268-1279.

6- Linde' $\mathrm{n} \mathrm{C,} \mathrm{Alm} \mathrm{A.} \mathrm{Prostaglandin} \mathrm{analogues} \mathrm{in} \mathrm{the}$ treatment of glaucoma. Drugs Aging. 1999;14(5):387398.

7- Berrino E, Supuran CT. Rho-kinase inhibitors in the management of glaucoma. Expert Opin Ther Pat. 2019 Oct;29(10):817-827.

8- Schehlein EM, Robin AL. Rho-Associated Kinase Inhibitors: Evolving Strategies in Glaucoma Treatment. Drugs. 2019 Jul;79(10):1031-1036.

9- Honjo, M, Inatani, M, Kido, N, Sawamura, T, Yue, BY, Honda, Y, et al. A myosin light chain kinase inhibitor, ML-9, lowers the intraocular pressure in rabbit eyes. Exp. Eye Res. 2002;75(2):135-142.

10-Hirata A, Inatani M, Inomata Y, Yonemura N, Kawaji T, Honjo M, et al. Y-27632, a Rho- associated protein kinase inhibitor, attenuates neuronal cell death after transient retinal ischemia. Graefes Arch Clin Exp Ophthalmol 2008;246(1):51-59.

11-Sugiyama T, Shibata M, Kajiura S, Okuno T, Tonari M, 
Oku H, et al. Effects of fasudil, a rho-associated protein kinase inhibitor, on optic nerve head blood flow in rabbits. Invest Ophthalmol Vis Sci. 2011;52(1): 64-69.

12-Bill A, Phillips CI. Uveoscleral drainage of aqueous humor in human eyes. Exp Eye Res. 1971;12(3):275281.

13-Zhou EH, Krishnan R, Stamer WD, Perkumas KM, Rajendran K, Nabhan JF, et al. Mechanical responsiveness of the endothelial cell of Schlemm's canal: scope, variability and its potential role in controlling aqueous humour outflow. $J R$ Soc Interface. 2012;9(71):1144-1155.

14-Gabelt BT, Kaufmann PL. Changes in aqueous humor dynamics with age and glaucoma. Prog Retin Eye Res. 2005;24(5):612-37.

15-Wiederholt M, Thieme H, Stumpff F. The regulation of trabecular meshwork and ciliary muscle contractility. Prog Retin Eye Res. 2000;19(3):271-295.

16-De Kater AW, Shahsafaei A, Epstein DL. Localization of smooth muscle and nonmuscle actin isoforms in the human aqueous outflow pathway. Invest Ophthalmol Vis Sci. 1992;33(2):424-429.

17-Wiederholt M. Direct involvement of trabecular meshwork in the regulation of aqueous humor outflow. Curr Opin Ophthalmol. 1998;9(2):46-49.

18-Rao PV, Deng P, Sasaki Y, Epstein DL. Regulation of myosin light chain phosphorylation in the trabecular meshwork: role in aqueous humor outflow facility. Exp Eye Res 2005;80(2):197-206.

19-Inatani M, Tanihara H, Katsuta H, Honjo M, Kido N, Honda Y. Transforming growth factor-beta 2 levels in aqueous humor of glaucomatous eyes. Graefes Arch Clin Exp Ophthalmol. 2001 Feb;239(2):109-13.

20-Ghanem AA, Elewa AM, Arafa LF. Endothelin-1 and nitric oxide levels in patients with glaucoma. Ophthalmic Res. 2011;46(2):98-102.

21-Pfitzer G. Invited review: regulation of myosin phosphorylation in smooth muscle. J Appl Physiol. 2001;91(1):32-39.

22-Liao JK, Seto M, Noma K. Rho kinase (ROCK) inhibitors. J Cardiovasc Pharmacol 2007;50(1):17-24.
23-Chircop M. Rho GTPases as regulators of mitosis and cytokinesis in mammalian cells. Small GTPases. 2014;5:e29770.

24- Loirand G. Rho Kinases in Health and Disease: From Basic Science to Translational Research. Pharmacological Reviews. 2015;67(4):1074-95..

25-Rath N, Olson MF. Rho - associated kinases in tumorigenesis: re - considering ROCK inhibition for cancer therapy. EMBO reports. 2012;13(10):900-8..

26-Honjo M, Inatani M, Kido N, Sawamura T, Yue BY, Honda $\mathrm{Y}$, et al. Effects of protein kinase inhibitor, HA1077, on intraocular pressure and outflow facility in rabbit eyes. Arch Ophthalmol. 2001;119(8):1171-8.

27-Stamer W, Braakman S, Zhou E, et al. Biomechanics of Schlemm's canal endothelium and intraocular pressure reduction. Progress in Retinal and Eye Research. 2014;44:86-98. .

28-Ren R, Li G, Le TD, et al. Netarsudil Increases Outflow Facility in Human Eyes Through Multiple Mechanisms. Invest Ophthalmol Vis Sci. 2016;57(14):6197-209..

29-Garnock-Jones KP. Ripasudil: first global approval. Drugs. 2014;74(18):2211-5..

30-Moshirfar M, Parker L, Birdsong OC, Ronquillo YC, Hofstedt D, Shah TJ, Gomez AT, Hoopes PCS. Use of Rho kinase Inhibitors in Ophthalmology: A Review of the Literature. Med Hypothesis Discov Innov Ophthalmol. 2018 Fall;7(3):101-111.

31-Moloney G, Petsoglou C, Ball M, Kerdraon Y, Hollhumer R, Spiteri N, Beheregaray S, Hampson J, D’Souza M, Devasahayam RN. Descemetorhexis without grafting for fuchs endothelial dystrophy-supplementation with topical ripasudil. Cornea. 2017;36:642-8.

32-Tanihara $\mathrm{H}$, Inoue $\mathrm{T}$, Yamamoto $\mathrm{T}$, et al. Additive Intraocular Pressure-Lowering Effects of the Rho Kinase Inhibitor Ripasudil (K-115) Combined With Timolol or Latanoprost: A Report of 2 Randomized Clinical Trials. JAMA Ophthalmol. 2015;133(7):755-61..

33-Sato S, Hirooka K, Nitta E, et al. Additive Intraocular Pressure Lowering Effects of the Rho Kinase Inhibitor, Ripasudil in Glaucoma Patients Not Able to Obtain 
Adequate Control After Other Maximal Tolerated Medical Therapy. Adv Ther. 2016;33(9):1628-34.

34-Yamada H, Yoneda M, Inaguma S, et al. A RhoAssociated Kinase Inhibitor Protects Permeability in a Cell Culture Model of Ocular Disease, and Reduces Aqueous Flare in Anterior Uveitis. J Ocul Pharmacol Ther. 2017;33(3):176-85.

35-Yasuda M, Takayama K, Kanda T, et al. Comparison of intraocular pressure-lowering effects of ripasudil hydrochloride hydrate for inflammatory and corticosteroid-induced ocular hypertension. PLoS One. 2017;12(10):e0185305.

36-Yamamoto K, Maruyama K, Himori N, Omodaka K, Yokoyama Y, Shiga Y, Morin R, Nakazawa T. The novel Rho kinase (ROCK) inhibitor K-115: a new candidate drug for neuroprotective treatment in glaucoma. Invest Ophthalmol Vis Sci. 2014;55:7126-36..

37-PubChem OPEN CHEMISTRY Database. Netarsudil USA: National Center for Biotechnology Information; 2018. [ [updated 2018; cited 2018]]. Compound Summary for CID $66599893 . \quad$ Available from: https://pubchem.ncbi.nlm.nih.gov/compound/Netars udil.

38-Lin CW, Sherman B, Moore LA, Laethem CL, Lu DW, Pattabiraman PP, et al. Discovery and Preclinical Development of Netarsudil, a Novel Ocular Hypotensive Agent for the Treatment of Glaucoma. J Ocul Pharmacol Ther. 2018;34(1-2):40-51.

39-Bacharach J, Dubiner HB, Levy B, et al. Double-masked, randomized, dose-response study of AR-13324 versus latanoprost in patients with elevated intraocular pressure. Ophthalmology. 2015;122(2):302-7..

40-Lewis R a, Levy B, Ramirez N, C Kopczynski C, Usner DW, Novack GD (2015) Fixed-dose combination of AR13324 and latanoprost: a double-masked, 28-day, randomised, controlled study in patients with open-angle glaucoma or ocular hypertension. $\mathrm{Br} \mathrm{J}$ Ophthalmol Bjophthalmol 2015:306778..

41-Lewis RA, Levy B, Ramirez N, et al. Fixed-dose combination of AR-13324 and latanoprost: a doublemasked, 28-day, randomised, controlled study in patients with open-angle glaucoma or ocular hypertension. $\mathrm{Br} \mathrm{J}$ Ophthalmol. 2016;100(3):339-44.

42-deLong MA, Sturdivant JM, Royalty SM, et al. Discovery and in vitro SAR of AR-12286, a Potent Kinase Inhibitor for the Treatment of Glaucoma. Investigative Ophthalmology \& Visual Science. 2009;50(13):4058.

43- Kopczynski C, Novack GD, Swearingen D, van Haarlem T. Ocular hypotensive efficacy, safety and systemic absorption of AR-12286 ophthalmic solution in normal volunteers. Br J Ophthalmol. 2013;97(5):567-72.

44-Williams RD, Novack GD, van Haarlem T, et al. Ocular hypotensive effect of the Rho kinase inhibitor AR-12286 in patients with glaucoma and ocular hypertension. Am J Ophthalmol. 2011;152(5):834-41.

45-Tanihara $\mathrm{H}$, Inatani $\mathrm{M}$, Honjo $\mathrm{M}$, et al. Intraocular pressure-lowering effects and safety of topical administration of a selective ROCK inhibitor, SNJ-1656, in healthy volunteers. Arch Ophthalmol. 2008;126(3):309-15.

46-Williams RD, Novack GD, van Haarlem T, Kopczynski C. Ocular hypotensive effect of the Rho kinase inhibitor AR-12286 in patients with glaucoma and ocular hypertension. Am J Ophthalmol. 2011;152:834-41.e1..

47-Honjo M, Tanihara H, Kameda T, Kawaji T, Yoshimura N, Araie M. Poten- tial role of Rho-associated protein kinase inhibitor Y-27632 in glaucoma filtration surgery. Invest Ophthalmol Vis Sci. 2007;48(12):5549-5557.

48-Van de Velde S, De Groef L, Stalmans I, et al. Towards axonal regeneration and neuroprotection in glaucoma: Rho kinase inhibitors as promising therapeutics. Prog Neurobiol. 2015;131:105-19.

49-Goldhagen B, Proia AD, Epstein DL, Rao PV. Elevated levels of RhoA in the optic nerve head of human eyes with glaucoma. J Glaucoma. 2012;21(8):530-8.

50-Meyer-ter-Vehn T, Sieprath S, Katzenberger B, Gebhardt S, Grehn F, Schlunck G. Contractility as a prerequisite for TGF-beta-induced myofibroblast transdifferentiation in human tenon fibroblasts. Invest Ophthalmol Vis Sci. 2006;47(11):4895-4904.

51-Arnold JJ, Hansen MS, Gorman GS, Inoue T, Rao V, Spellen S, et al. The effect of Rho-associated kinase 
inhibition on the ocular penetration of timolol maleate. Invest Ophthalmol Vis Sci 2013; 7;54(2):1118-26.

52-Bacharach J, Dubiner HB2, Levy B3, Kopczynski CC3, Novack GD4; AR-13324-CS202 Study Group. Doublemasked, randomized, dose-response study of AR-13324 versus latanoprost in patients with elevated intraocular pressure. Ophthalmology. 2015;122(2):302-7

53-Tanihara H, Inoue T, Yamamoto T, Kuwayama Y, Abe H, Araie M; K-115 Clinical Study Group. Phase 2 randomized clinical study of a Rho kinase inhibitor, K115 , in primary open-angle glaucoma and ocular hypertension. Am J Ophthalmol. 2013 Oct;156(4):731-6. 